\title{
Servicio de Anestesiología del Hospital de Urgencia Asistencia Pública: ¿Quiénes somos?
}

\author{
Anesthesia in Asistencia Pública Hospital. Who are we? \\ Guillermo Reyes Guzmán'
}

\begin{abstract}
The Asistencia Pública was founded on August 7, 1911. It was conceived as a public hospital focused on emergency medicine. Its first location was a building acquired from the Franciscan community located at $\mathrm{N}^{\circ}$ 85 San Francisco street, at the intersection with San Carlos street (later renamed as Alonso Ovalle). A new building was inaugurated on December 15, 1967 and its infrastructure allowed the development of various services and units that modernized, expanded and improved emergency patient care. Recently, a new facility was incorporated, called Torre Valech, inaugurated in February 2019, housing modern facilities for the emergency service, the surgical pavilions and the intensive care unit. The year 1936 marked a milestone for the Asistencia Publica Anesthesiology, due to the work of Ernesto Frías, MD, specialist trained in United States and Argentina, who organized for the first time an annual anesthesia course, working on an Ad Honorem basis. Nevertheless, the true founder of the Anesthesiology Service and Intensive Care Unit of the José Joaquín Aguirre Hospital, was Dr. Max Arriagada Loyola. He was also a pioneer in allowing medical students to do the work of technical assistants in the specialty, during their undergraduate studies. During the eighties, the Asistencia Publica's Anesthesiology Service added new specialists who were filling the available positions both, during the daytime and on call hours. Incorporation of new technology also allowed to offer more complex and safer anesthesia procedures. Teaching has been a fundamental part of the activities of the service since its creation. First with of Dr. Arriagada's disciples, then, with the Ministerial Training Program for Anesthesiologists which in 1999 became the Postgraduate Program in Anesthesiology and Resuscitation of the Universidad de Santiago de Chile. In 2013 the program was accredited for the first time.

\section{RESUMEN}

La Asistencia Pública fue fundada el 7 de agosto de 1911, pensada como un servicio hospitalario público centrado en los pacientes de urgencia. Su primera ubicación fue un local adquirido a la comunidad franciscana ubicada en calle San Francisco N ${ }^{\circ} 85$ esquina de calle San Carlos (hoy Alonso Ovalle). El nuevo edificio fue inaugurado el 15 de diciembre de 1967 y su infraestructura permitió el desarrollo de varios servicios y unida-

Hospital de Urgencia Asistencia Pública.

Fecha de recepción: 16 de noviembre de 2020

Fecha de aceptación: 23 de noviembre de 2020

\section{ORCID}

0000-0003-1962-7658

Correspondencia:

Guillermo Reyes Guzmán

greyes.guzman49@gmail.com 
des que modernizaron, ampliaron y mejoraron la atención del paciente de urgencia. Finalmente, su última ampliación, la llamada Torre Valech, fue incorporada al edificio en febrero de 2019, albergando modernas instalaciones para la urgencia, los pabellones quirúrgicos y la Unidad de Tratamiento Intensivo. El año 1936 marcó un hito en la anestesiología de la Asistencia Pública, a partir del trabajo del doctor Ernesto Frías, especializado en Estado Unidos y Buenos Aires, quien organizó en forma ad honorem un curso anual de anestesia. Pero el verdadero fundador de un servicio de anestesiología y, a su vez, de la Unidad de Tratamiento Intensivo del hospital José Joaquín Aguirre, fue el Dr. Max Arriagada Loyola, quien fue iniciador de la docencia en pregrado, para que alumnos de medicina hicieral la labor de auxiliares técnicos en la especialidad. Durante la década de los 80, el desarrollo del Servicio de Anestesiología de la Posta Central significó la suma de nuevos especialistas que fueron ocupando los cargos disponibles, tanto en horario diurno como de residencia, y la incorporación de nueva tecnología que permitió ofrecer anestesias más complejas y más seguras. La docencia ha formado parte de las actividades del servicio desde su creación: primero con los discípulos del Dr. Arriagada, luego con el Programa de Formación Ministerial de Anestesiólogos y, finalmente, se convirtió en el Programa de Postgrado en Anestesiología y Reanimación de la Universidad de Santiago de Chile a partir del año 1999, acreditándose por primera vez en el año 2013.

\section{Introducción}

P ara realizar una reseña histórica del Servicio de Anestesiología del Hospital de Urgencia Asistencia Pública (ex Posta Central), es necesario recurrir a antiguas publicaciones y a documentación oficial del Ministerio de Salud, a notas de historiadores de nuestra medicina nacional y sudamericana y a diversas publicaciones de colegas que han formado parte de esa historia. Se ha recurrido también a notas de antiguos boletines de la policía de Santiago y a viejos artículos de la "Revista de la Beneficencia Pública", todos ellos de las primeras décadas del siglo pasado. Más recientemente, existe valiosa información en la "Revista de la Asistencia Pública", que se publicó en las décadas del 70 y 80, con una periodicidad semestral. Finalmente, existen datos aportados por viejos médicos y antiguos funcionarios del hospital, que han compartido sus recuerdos y experiencias[1].

Para entender mejor esta breve reseña del desarrollo de la anestesiología en el Hospital de Urgencia Asistencia Pública (HUAP), se debe tener en cuenta como telón de fondo el contexto social y político del Santiago de fines del siglo XIX y comienzos del XX.

En aquel período Chile atravesaba por una época política compleja, con lucha de poderes y un parlamentarismo que predominaba en las decisiones públicas, manejadas principalmente por una aristocracia en la cual destacaban varios médicos, lo cual podía suponer al menos teóricamente, cambios y mejoras en la medicina del país, entonces bastante precaria y deficitaria. Sin embargo, lo anterior no correspondía a esa realidad. Ejemplo de ello es la existencia de un grupo de médicos pioneros interesados en crear un nuevo hospital dedicado a la patología de urgencia, idea revolucionaria, que se enfrentaría a todo tipo de dificultades, ya que ésta no fue considerada entre las prioridades de entonces ni del futuro próximo[2].

Superando todos esos obstáculos la Asistencia Pública fue fundada el 7 de agosto de 1911, siendo pensada como una organización y estructura novedosa para la época: un servicio hospitalario público centrado en los pacientes de urgencia, un concepto que como ya se mencionó, no se consideraba importante ni en Chile ni en Latinoamérica de ese entonces.

\section{La atención médica de urgencia antes de la Asistencia Pública}

Hasta ese año, los pacientes de urgencia, los traumatizados y los heridos graves debían consultar a alguno de los hospitales generales existentes o intentar conseguir la visita de un médico a domicilio. Los pacientes accidentados en la vía pública, eran trasladados en cualquier medio de transporte a alguno de estos hospitales o a su domicilio y los más graves en coches tirados por caballos de la policía municipal, hasta los cuarteles de la propia policía. Estos coches eran los mismos en que se trasladaba a los ebrios detenidos (Figura 1)[12].

En estos cuarteles policiales los enfermos eran atendidos muy precariamente, por estudiantes de medicina y de enfermería contratados para tales efectos, los cuales poco podían hacer con los escasos recursos disponibles en un recinto no apto para la atención de salud. En los casos más graves se intentaba enviar a los pacientes a algún hospital en estos mismos coches que, como ya se ha insinuado, distaban mucho de tener las mínimas condiciones de comodidad y seguridad. Evidentemente, en tales condiciones, la mortalidad era inevitablemente elevada. Esta forma 
de atención se llamó "Servicio Sanitario y Asistencia Pública de la Policía de Santiago" y en las comunicaciones oficiales se le designó sencillamente como "Asistencia Pública"[2].

Desde 1880 en adelante, existió una gran presión política y social para mejorar la atención médica y en especial la de urgencia. Se crearon numerosas comisiones conformadas por importantes médicos de la época y el tema fue motivo de discusión política en la Universidad de Chile y en el Congreso Nacional. No obstante, en el corto plazo no se lograron avances concretos. La tarea de concientizar a las autoridades fue prolongada, dura y muchas veces incomprendida, siendo sostenida y promovida por varios destacados médicos de la época, entre los que se encuentran los doctores Germán Valenzuela Basterrica, José Joaquín Aguirre, Alcibíades Vicencio y Ricardo Ávila. Sin embargo, dentro de este grupo de profesionales, sobresale el nombre del doctor Alejandro del Río SotoAguilar quien, fruto de esta larga lucha política, social y administrativa consiguió, finalmente, que el Estado de Chile se involucrara en el tema, comenzando por comprar una gran casona perteneciente a la comunidad religiosa franciscana en la calle San Francisco $\mathrm{N}^{\circ}$ 85 esquina de calle San Carlos (hoy Alonso Ovalle) (Figura 2). Después de la adquisición de este recinto destinado a albergar a la Asistencia Pública, se enfrentó otra gigantesca tarea para conseguir los recursos económicos necesarios para su implementación y puesta en marcha[2].

Por aquellos años, el concepto de "Asistencia Pública" era sinónimo de "Beneficencia Pública", pero para la época de la fundación de lo que actualmente es nuestro hospital (uno más de lo que fue posteriormente sectorizado en diferentes zonas de Santiago), el concepto ya había ido cambiando, comenzando a entenderse como una institución hospitalaria de servicio público, enfocada en la atención de los pacientes de urgencia durante las 24 horas del día y todos los días de la semana, concepto absolutamente novedoso hasta entonces en el país.

\section{Los comienzos}

En pocos meses, el edificio de San Francisco No 85 fue refaccionado y habilitado, en gran medida gracias al tesón de sus promotores y en particular el del Dr. Alejandro del Río, quien enfrentó no pocas dificultades. La inauguración se realizó el 07 de agosto de 1911, con una solemne ceremonia, presidida por el Ministro del Interior, Rafael Orrego, en representación del Presidente de la República Don Ramón Barros
Luco, ausente por enfermedad. A ella asistieron altas autoridades de gobierno, autoridades de salud y de la beneficencia pública, así como personalidades de la sociedad capitalina. En el ambiente de la inauguración se percibía una rara mezcla de curiosidad e incredulidad. La prensa capitalina, sin embargo, celebró unánimemente el acontecimiento, declarando que la población podría ahora sentirse protegida y que su salud estaría garantizada como nunca antes[5].

La importancia de la puesta en marcha de la Asistencia Pública, también fue reconocida internacionalmente. El eminente profesor de cirugía Émile Widal la visitó desde París a dos meses de su inauguración y declaró que, por su organización y funcionamiento, nada tenía que envidiarle a los mejores servicios de urgencia de Francia[1].

La organización con que su primer director, el Dr. Alejandro del Río (quien ostentó este cargo por 18 años) concibió y puso en marcha fue tan visionaria, que no obstante el correr del tiempo, mantuvo dicha

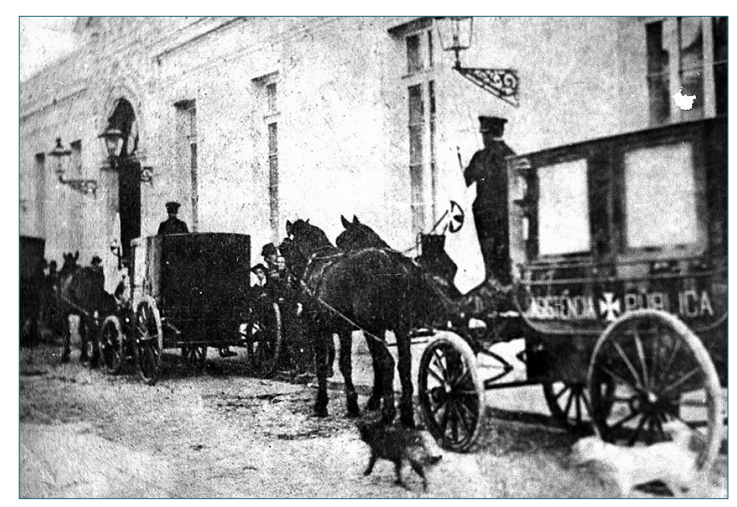

Figura 1. Coches-ambulancia de la Asistencia Pública a principios del siglo XX.

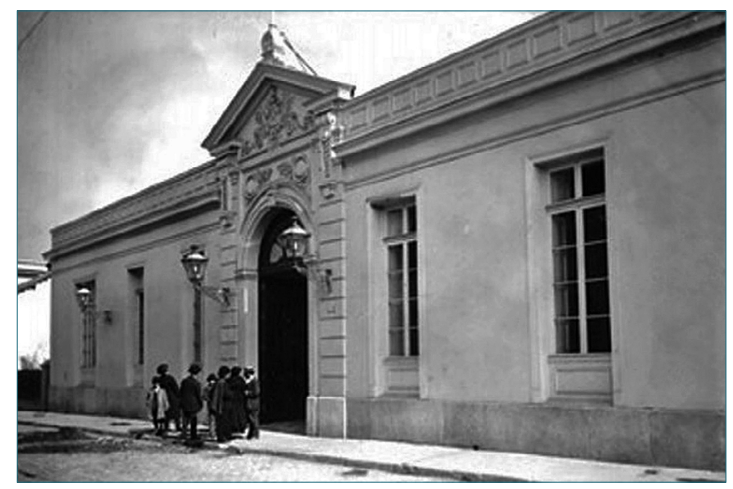

Figura 2. La vieja casona de San Francisco 85, primera sede de la Asistencia Pública, fundada el 7 de agosto de 1911. 
estructura y organización casi sin modificaciones por más de 30 años con notable eficiencia[1]. La Asistencia Pública fue hasta 1938, cuando se inauguró la Clínica Santa María, el único servicio de urgencia de Chile.

La Asistencia Pública en sus comienzos contaba con cuarenta y siete funcionarios en seis turnos rotativos que consideraban cuatro médicos, que cubrían las veinticuatro horas del día por período de dos horas y media cada uno, y una noche cada seis días. A ellos se sumaba una enfermera, personal paramédico, auxiliares, practicantes y personal administrativo. Además, era habitual que se sumaran a cada turno, un número variable de estudiantes de medicina y enfermería. Al menos uno de los médicos de turno, debía estar "entrenado" en administrar las anestesias que fuesen necesarias[8]. El Dr. Alejandro del Río, creó una escuela de auxiliares, para no depender exclusivamente de las monjas que atendían en otros hospitales.

Durante los primeros quince años, se mantuvo también un sistema de "atención domiciliaria de urgencias nocturnas". Un médico por turno debía acudir, transportado por un cochero, a los llamados de pacientes de urgencia. Estas atenciones tenían un muy bajo costo para los pacientes y eran gratuitas para los menesterosos. En la práctica, casi nadie las pagaba[6].

Hacia 1922 se inauguró la "Botica de Urgencia", anexa al edificio central, la cual también funcionaba las veinticuatro horas del día, convirtiéndose por esos días en el centro farmacéutico mejor abastecido del país. Al año siguiente el Dr. del Río organizó la construcción, en la misma casona de un segundo piso, donde se ubicaron oficinas administrativas, archivos y salas de reuniones (Figura 3).

\section{Las primeras anestesias en la era de la nueva Asistencia Pública}

A fines del siglo XIX y comienzos del siglo XX en la mayoría de los hospitales de Chile se realizaban procedimientos quirúrgicos acotados, sin anestesia[12].

La anestesia comenzó a utilizarse en el mundo a mediados del siglo XIX, pero en Chile y en toda Sudamérica tardó mucho más en instituirse como procedimiento médico habitual. Como una situación anecdótica, el diario El Mercurio de Valparaíso publicó el 23 de octubre de 1848 un reportaje sobre "La Primera Anestesia General por Inhalación en Chile", en relación a una cirugía realizada en el Hospital San Juan de Dios de este mismo puerto (hoy Hospital Carlos van Buren) (Figura 4), donde se utilizó cloroformo en una paciente de 90 años, para una amputación de extremidad superior, realizada por el cirujano Dr. Francisco Villanueva, procedimiento que concluyó con éxito. Este evento ocurrió solo dos años después de que William Thomas Morton realizara la primera anestesia con éter en Boston en octubre de 1846. No existe registro del profesional porteño que realizó esta primera anestesia en Chile, no constando si habría sido el mismo cirujano o un joven colega cuyo nombre no pasó a la historia. Lo más curioso es que la siguiente anestesia de este tipo se administró en Chile recién veinte años después, en ese mismo hospital[7],[13].

El Dr. Héctor Venturini, en su monografía publicada en 2010 "Historia de la Anestesia en Sudamérica" escribe: "Comenzando el siglo XX en la mayoría de los pabellones de cirugía latinoamericanos, se dejaron de escuchar los gritos de los pacientes siendo operados. En adelante solo se escucharían los gritos de los cirujanos. No habría más suicidios de pacientes aterrorizados, que se negaban a ser operados. Los enfermos dejaron de ser sujetados violentamente, ni era necesario amarrarlos a las mesas operatorias. Nunca más se realizaron intervenciones contra la voluntad del paciente. El siglo XX en Sudamérica trajo un cambio fundamental después de una vasta y triste etapa ancestral de intervenciones quirúrgicas horrorosamente dolorosas"[14].

El Dr. Venturini finaliza su reseña casi poéticamente, señalando: "Gracias a la anestesia, el sol del naciente siglo XX iluminó las salas de cirugía". Por lo menos en Chile y en la Asistencia Pública, así fue[14].

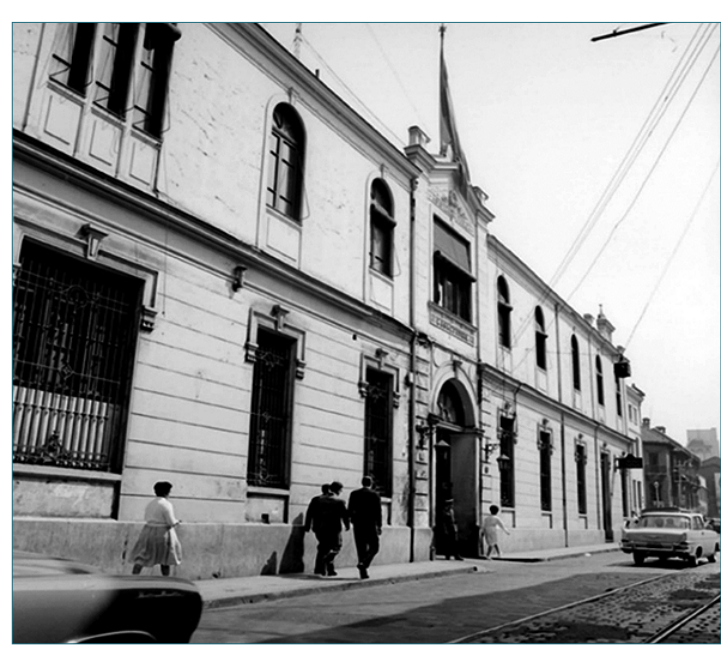

Figura 3. Edificio de la Asistencia Pública después de sus ampliaciones realizadas en 1922 y 1923. 


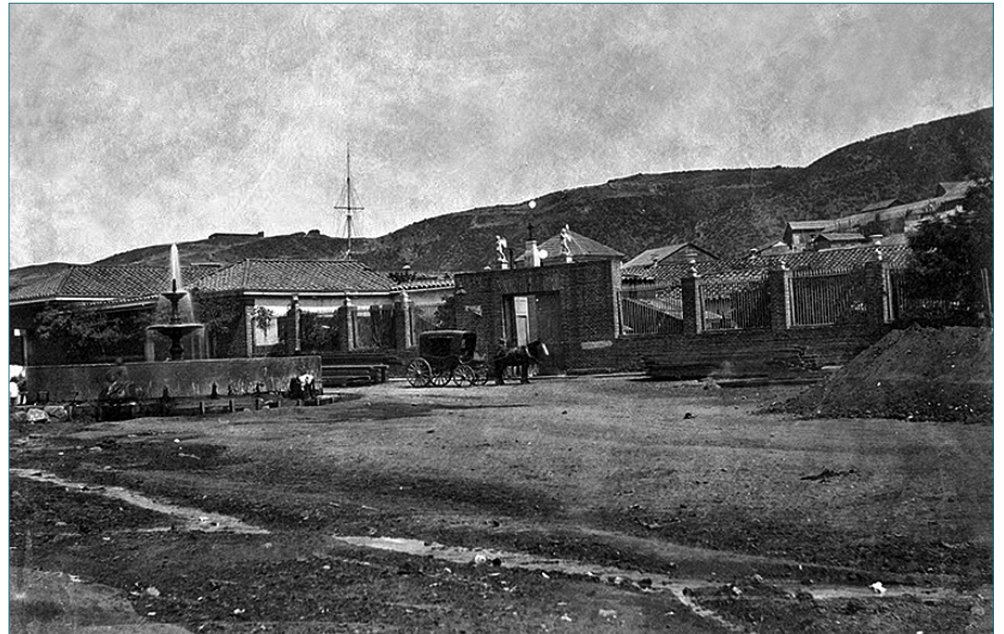

Figura 4. Hospital San Juan de Dios de Valparaíso, donde en 1848 se realizara la primera anestesia general en Chile.

\section{Las primeras anestesias en la Asistencia Pública}

Los primeros "Anestesistas" o "Anestesiadores" de la Asistencia Pública, fueron estudiantes de medicina o médicos jóvenes, medianamente entrenados en el manejo del éter y del cloroformo. Para su administración se utilizaban mascarillas faciales de alambre cubiertas de gasas, sobre las que se goteaba el agente anestésico hasta lograr la inconciencia del paciente, y en algunos casos parcialmente la del operador. En paralelo, se daba inicio a la administración de las primeras y rudimentarias anestesias raquídeas y a precarios manejos del opio, la codeína, la escopolamina y, más adelante, la morfina intravenosa.

Según descripciones de la época, con bastante frecuencia los primeros actos anestésicos se complementaban con bebidas alcohólicas, entre ellas vino, ron, cerveza y gin por vía oral, con el consiguiente riesgo de aspiración y sus catastróficas consecuencias. Además, eran parte fundamental del equipo quirúrgico, robustos mocetones que contenían y amarraban a los pacientes[17]. Posteriormente, a partir de la segunda década del siglo XX, ya no se amarraba a los pacientes; de alguna forma se los sedaba. Además, con anestesia local se realizaban intervenciones quirúrgicas impensadas en estos tiempos.

En un artículo de los Archivos de La Sociedad Chilena de Cirugía de 1972, el Dr. Ignacio González Ginouvés (jefe de un turno de la Asistencia Pública en el año 1925) recordada la introducción del "moderno aparato de Ombredanne", para administrar anestesias por inhalación (Figura 5). Este rudimentario aparato inventado en Europa por el año 1908, era un gran avance a esa fecha. "Estaba a cargo de un interno de medicina o de un médico recién recibido, entrenado en su manipulación. A su llegada al turno, recibía breves y nerviosas instrucciones de otro interno que ya terminaba su turno", señalaría el Dr. González[3]. Este colega, destacaría más tarde, como decano de la Facultad de Medicina de la Universidad de Concepción (1948-1955), Jefe de Cirugía del Hospital Salvador (1956-1962) y Rector de la Universidad de Concepción (1962-1968). En contrapartida, cuando se trataba de afecciones infraumbilicales, se solía preferir la anestesia raquídea, técnica de anestesia regional que algunos trataban de dominar[3],[15].

No se debe dejar de mencionar que en las anestesias de entonces también participaba un cierto número de monjas francesas entrenadas en su país y religiosas de la congregación de San Vicente.

En paralelo en estos años, se estaban habilitando otros servicios de urgencia más pequeños y menos dotados, distribuidos en diferentes comunas de Santiago y que fueron conocidos como "postas", por la sucesión de médicos en turno que atendía en estos recintos: la Posta 2 en calle Ñuble, la Posta 3 en calle Chacabuco y la Posta 4 en calle Villaseca. Bajo el mismo concepto, la Asistencia Pública de calle San Francisco fue denominada la Posta Central.

\section{La labor del Dr. Ernesto Frías en el desarrollo de la anestesia de urgencia}

El año 1936 marcó un hito en la anestesiología de la Asistencia Pública, a partir del trabajo del doctor Ernesto Frías (Figura 6), especializado en anestesiología en Estado Unidos y Buenos Aires, quien organizó 
en forma ad honorem un curso anual de anestesia, en el Hospital del Salvador, orientado principalmente a médicos y enfermeras de urgencia. Para ello, el Drs. Frías contó con la valiosa ayuda de los Drs. Luis Aguilar y Félix de Amesti. Ellos popularizaron en la Asistencia Pública, y posteriormente, en otros hospitales de Santiago, la administración de anestesia inhalatoria con el aparato de Ombredanne, creado para la administración inhalatoria de éter. Se utilizó también por primera vez el óxido nitroso y el ciclopropano, y se introdujo el uso del cloroformo, la intubación traqueal y el circuito cerrado de anestesia.

El Dr. Frías diseñó la Chilean Midget, una máquina de anestesia simplificada, pensada para las condiciones de un país de pocos recursos como Chile y comercializada por la empresa Foregger. Era una máquina fácilmente desplazable, muy apta para el país entre los años 1935-1938, que actualmente se expone en el Museo de Historia de la Medicina de la Universidad de Chile. En 1941 el Dr. Frías se integró al Servicio de Cirugía del Hospital del Salvador como Jefe del Departamento de Anestesiología y en 1946 fue uno de los miembros fundadores de la Sociedad de Anestesiología de Chile. Aunque hasta su muerte en Houston el 20 de junio de 1977 no se había creado tal distinción, por lejos mereció ser el primer Maestro de la Anestesiología Chilena, reconocimiento que finalmente recayó en uno de sus discípulos, el Prof. Luis Cabreras[15],[16].

El Dr. Luis Aguilar, director de la Asistencia Pública y jefe de Servicio de Cirugía del Hospital San Borja, brindó apoyo a los inseguros comienzos de la anestesiología en la Asistencia Pública, siendo la primera institución hospitalaria que contó con un "moderno" aparato de anestesia con fuente de gas[17].

\section{La anestesia en las catástrofes}

En enero de 1939, ocurrió un gran terremoto en la ciudad de Chillán, catástrofe a la cual la Asistencia Pública prontamente envió una delegación de auxilio. Concurrieron a Chillán veintidós médicos, ocho enfermeras, auxiliares paramédicos de planta y voluntarios y varios miembros de la Cruz Roja. En este grupo iban dos médicos jóvenes y una enfermera entrenados en anestesia por el Dr. Frías.

Un avión servía de puente entre Chillán y Santiago, trasladando a la capital los pacientes más graves y el instrumental, medicamentos, ropa y otros insumos hacia la zona de la tragedia. En paralelo, dos veces al día un servicio de trenes complementaba este servicio.

Con este recurso humano y logístico fueron atendidos, durante doce días de incesante labor, mil

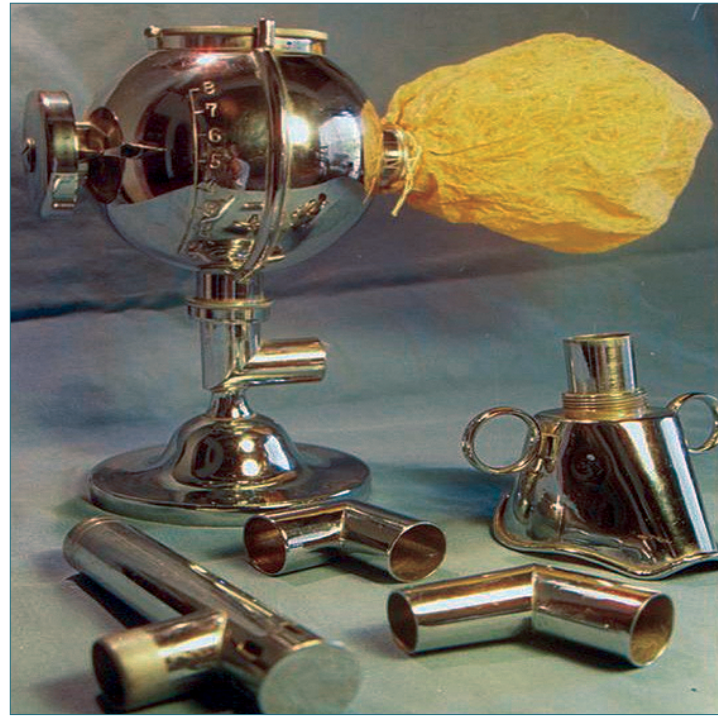

Figura 5. Aparato de Ombredanne.

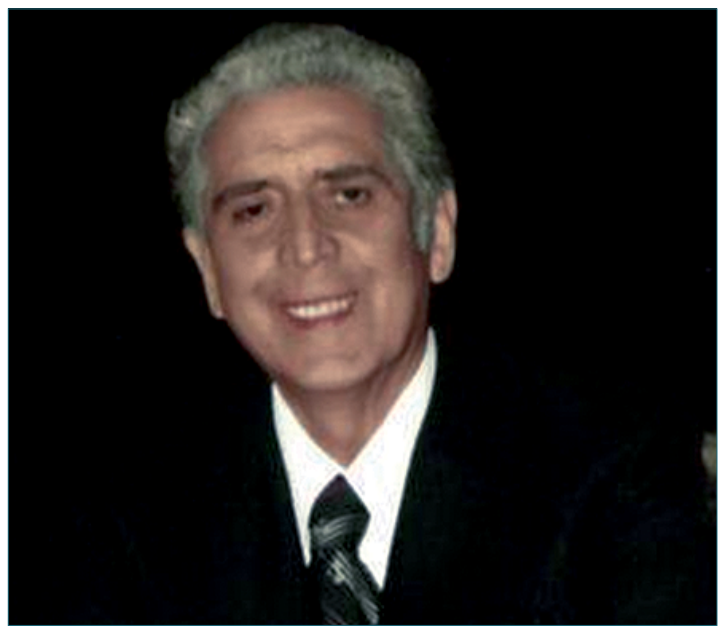

Figura 6. Dr. Ernesto Frías Meneses (1908-1977).

doscientos ochenta y cuatro heridos, cuatrocientos veintinueve fracturados y numerosas otras lesiones menores. Se estima que durante el operativo se administraron más de cien anestesias generales y más de trescientas anestesias raquídeas.

Esta no es la única oportunidad en que la Asistencia Pública ha colaborado con operativos de salud relacionados con catástrofes, existiendo casos en los cuales incluso se ha ayudado más allá de las fronteras, apoyando a países hermanos en situaciones difíciles. Ejemplo de ellos son las misiones enviadas a[8]:

- 1947: Terremoto en San Juan, Argentina. 
- 1949: Terremoto en Ambato, Ecuador.

- 1952: Guerra civil en La Paz, Bolivia.

- 1967: Terremoto en Caracas, Venezuela.

Coincidentemente, el Dr. Alejandro del Río falleció pocos días después de concluir el operativo que llevó ayuda a Chillán, el 5 de febrero de 1939. La Junta de Beneficencia que entonces administraba la Asistencia Pública acordó dar su nombre a la institución, la cual a partir de ese momento pasó a llamarse "Asistencia Pública Dr. Alejandro del Río" (Figura 7).

\section{La anestesia y los "auxiliares técnicos"}

A principios de la década de los 60, se hizo evidente que la infraestructura hospitalaria era muy deficitaria en Santiago, en especial la de urgencia. El entonces director de la Asistencia Pública, Dr. Mariano Bahamondes, encabezó una larga e intensa campaña para modernizar y mejorar la institución, que todavía funcionaba en la vieja casona de calle San Francisco. En esta cruzada, lo acompañaron numerosos y destacados médicos, políticos y filántropos de la época. Algunos medios, compararon este empuje y determinación con el "espíritu Alejandro del Río".

Uno de los proyectos emblemáticos consideró dotar a la Institución de especialistas, entre ellos anestesiólogos, por entonces muy escasos. En tal sentido y como ejemplo de este déficit y de los denodados esfuerzos por cubrir lo mejor posible la atención anestésica de urgencia, un "INSTRUCTIVO DEL MINISTERIO DE SALUD" del 07 de octubre de 1976, señalaba en su artículo 43: "Mientras no exista el número suficiente de anestesiólogos en el país, los servicios de urgencia tendrán un solo profesional funcionario de esa especialidad, el que desempeñará sus funciones en un turno. Además, tendrá bajo su responsabilidad la organización y el funcionamiento adecuado de la anestesia de los demás turnos, con "auxiliares técnicos", personal de colaboración médica u otros, debidamente entrenados que se desempeñarán bajo la tuición y supervigilancia directa del jefe de turno respectivo". Desde esa fecha, debieron pasar muchos años para que cada turno contara con un médico especialista en anestesia[8].

\section{El nuevo edificio de Portugal 125}

Los esfuerzos para mejorar la atención de urgencia se vieron reflejados el año 1965 en la aprobación de la construcción del nuevo edificio de la Asistencia Pública, ubicado en calle Portugal 125, lugar donde hasta el día de hoy se emplaza la institución (Figura

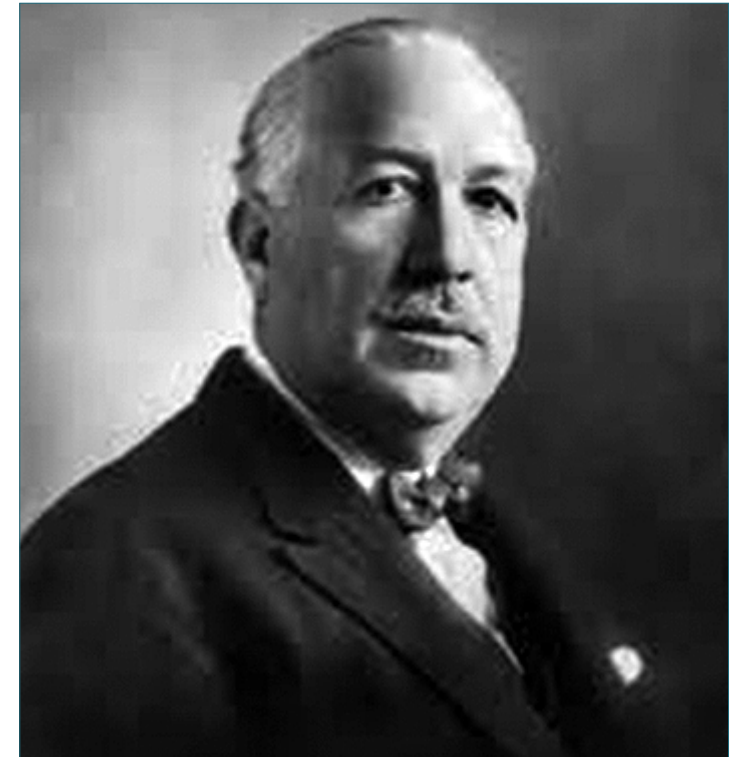

Figura 7. Dr. Alejandro del Río Soto-Aguilar (1967-1939).

8). El nuevo edificio fue inaugurado por el presidente Eduardo Frei Montalva el 15 de diciembre de 1967 y su infraestructura permitió el desarrollo de varios servicios y unidades que modernizaron, ampliaron y mejoraron la atención del paciente de urgencia (Figura 9). Dentro de estas mejoras se crearon progresivamente los Servicios de Quemados, Traumatología, Unidad de Cuidados Intensivos, Dental y Maxilofacial, Neurocirugía, Kinesiología, Radiología, Laboratorio Clínico y Banco de Sangre.

Otro de los avances relevantes que trajo aparejado la inauguración del nuevo edificio de la Asistencia Pública, fue la puesta en marcha de un helipuerto anexo al edificio, que permitió trasladar en forma oportuna a pacientes que habían sufrido accidentes en lugares lejanos al hospital. Este recurso significó por cierto un nuevo desafío tanto para los cirujanos como para los anestesistas, puesto que implicó la hospitalización de pacientes politraumatizados con mayor nivel de gravedad (Figura 10)[9].

Debe destacarse también que el nuevo edificio contaba con una sala de recuperación anestésica, atendida por enfermeras universitarias, a cargo de las indicaciones del anestesista, condición no habitual en otros hospitales de Santiago.

\section{Max, sus "enanos" y su motoneta}

Entre mediados de los años 60 y los 70 se desempeñaron en la Asistencia Pública los llamados "auxi- 


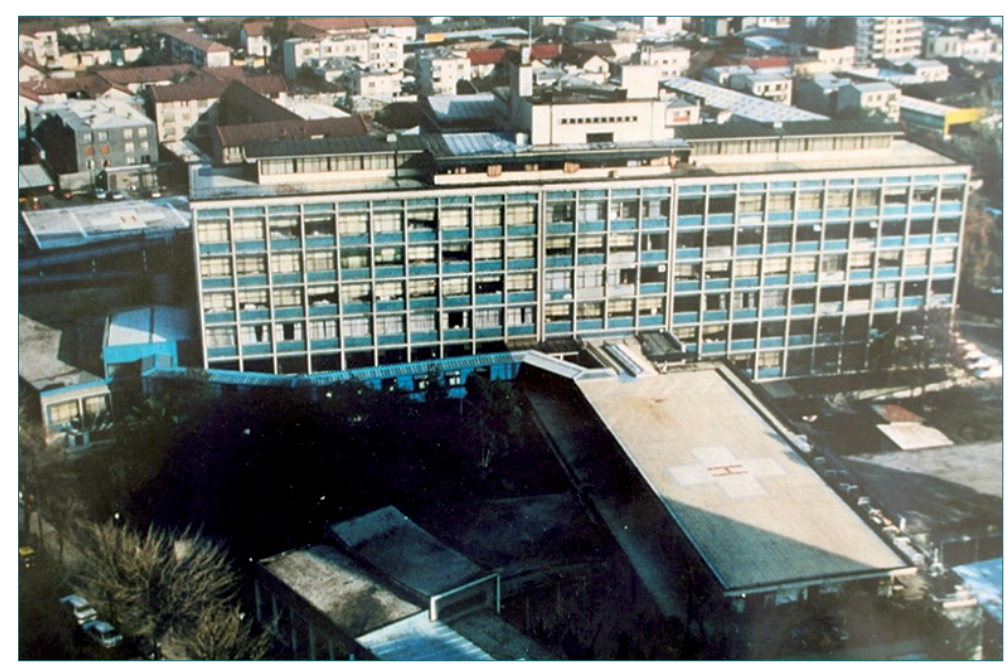

Figura 8. "Nuevo" edificio de la Asistencia Pública, inaugurado el 15 de diciembre de 1967. liares técnicos de anestesia" de acuerdo al instructivo ministerial antes mencionado. Éstos eran alumnos de sexto y séptimo año de medicina, que recibían un entrenamiento teórico práctico en anestesia y reanimación. Ellos realizaban los turnos que no se encontraban cubiertos por médicos anestesiólogos. A la mayoría los entrenaba el Dr. Max Arriagada (Figura 11), primer jefe de la Unidad de Anestesiología del hospital. Por esta razón, se los Ilamó coloquialmente los "enanos de Max".

En aquella época, en que las becas de especialización no eran el mecanismo regular de formación de postgrado, el Dr. Arriagada era parte de aquellos anestesiólogos que, radicados en un hospital, se constituyeron en los maestros que recibieron y formaron a los futuros anestesistas del país. En este selecto grupo se encuentran, además de él, el Dr. Luis Cabrera en el Hospital del Salvador, el Dr. Felipe Olivarí en el Hospital José Joaquín Aguirre, el Dr. Óscar de Gatica en el Hospital San Juan de Dios y el Dr. Waldemar Badía en el Hospital Clínico de la Universidad Católica. Todos ellos han sido nombrados como "Maestros de la Anestesiología Chilena" y con toda certeza, no mediar su prematura muerte, el Dr. Max Arriagada habría sido distinguido de igual forma.

El Dr. Max Arriagada y el Dr. Manuel Pellicer eran los únicos médicos anestesiólogos en la Asistencia Pública a fines de los 60 y formaban parte cada uno de un turno, pero con frecuencia debían acudir a otras de las postas de Santiago cuando se requería anestesiar a un paciente en dichos recintos. Para tal efecto, el Dr. Max Arriagada contaba con una motoneta, donde transportaba un maletín con instrumental y

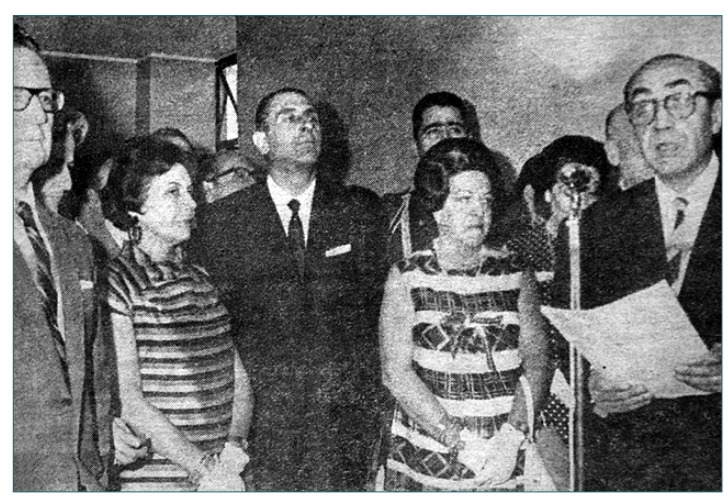

Figura 9. Presidente Eduardo Frei y presidente del Senado Salvador Allende en la ceremonia de inauguración del nuevo edificio de la Asistencia Pública Dr. Alejandro del Río $(15 / 12 / 1967)$

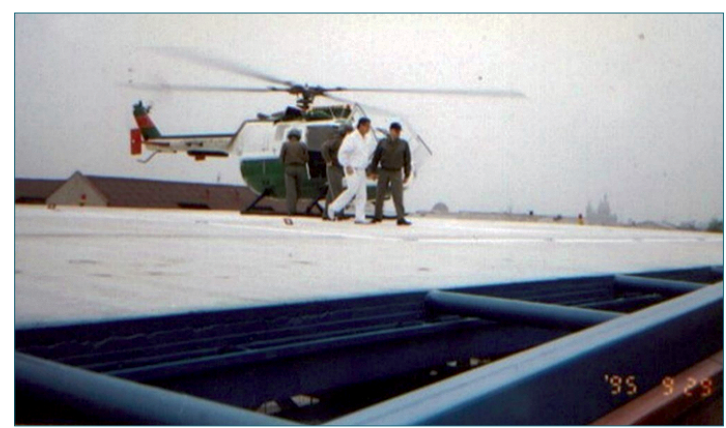

Figura 10. Helipuerto de la Asistencia Pública. 
medicamentos y a la arsenalera en el asiento trasero. Esta era la forma como en las otras postas se podían realizar las cirugías de los pacientes más graves, cuando estos no podían ser trasladados a otro recinto. Sin embargo, en la práctica, muchos pacientes eran anestesiados por un cirujano del turno que hacía las veces de anestesista, incluso muchas veces administrando una anestesia raquídea y luego operando al paciente. Una realidad que se vivió en los servicios de urgencia hasta los años 80.

A comienzos de los años 70, el Dr. Max Arriagada se autoexilió en Alemania, pero volvió pronto a Chile, donde según señaló fue recibido cálidamente por la que él llamaba "su posta." En el transcurso de la década y gracias a la gestión del Dr. Arriagada, quien por un lado había reclutado y por el otro había formado a nuevos especialistas, cada turno contaba ya con un anestesiólogo, con lo que se constituía un incipiente "Servicio de Anestesiología". Haciendo un corte en el tiempo, el año 1981, cada turno tenía un anestesista, algunos de la cantera de la Asistencia Pública y otros formados en otros servicios: los Drs. Michael Zanberck (primer turno), Ramón Danitz (segundo turno), Marcial Letelier (tercer turno), Guillermo Reyes (cuarto turno), Carlos Reyes (quinto turno) y Ricardo Bustamante (turno volante). En esa época ya los turnos eran de 6 horas de día (una mañana y una tarde) y una noche cada 6 días, cubriéndose la semana entera con especialistas. Posteriormente y cercano a fines de los 80, ya se habían incorporado a los diferentes turnos los Drs. Eliana Hidalgo, Soledad Covarrubias, Silvia Chicago, Víctor Hanna, Marco Guerrero, David Castro y Raúl Prieto.

Estos anestesistas contaban con cargos para su contratación, pero aquellos que se unían al servicio para formarse como especialistas no. Por lo tanto, estos últimos fueron contratados a través del Plan de Empleo Mínimo (PEM), iniciativa instituida por el gobierno de la época para absorber mano de obra y bajar los altos niveles de cesantía existentes a costa de sueldos que no se correspondían con la responsabilidad del cargo.

Durante las Fiestas Patrias de 1981 (17 de septiembre) en una celebración familiar, el Dr. Max Arriagada murió prematuramente a los 47 años, dejando sembrada la idea de formar anestesiólogos en conjunto con otros hospitales públicos, para reducir el déficit de especialistas en anestesiología que aquejaba al Sistema Público de Salud. Ese sueño fue concretado en los siguientes años, a través de la implementación de un programa de formación de anestesiólogos radicado en el Hospital de Urgencia Asistencia Pública: el Programa de Formación Ministerial de Anestesió-

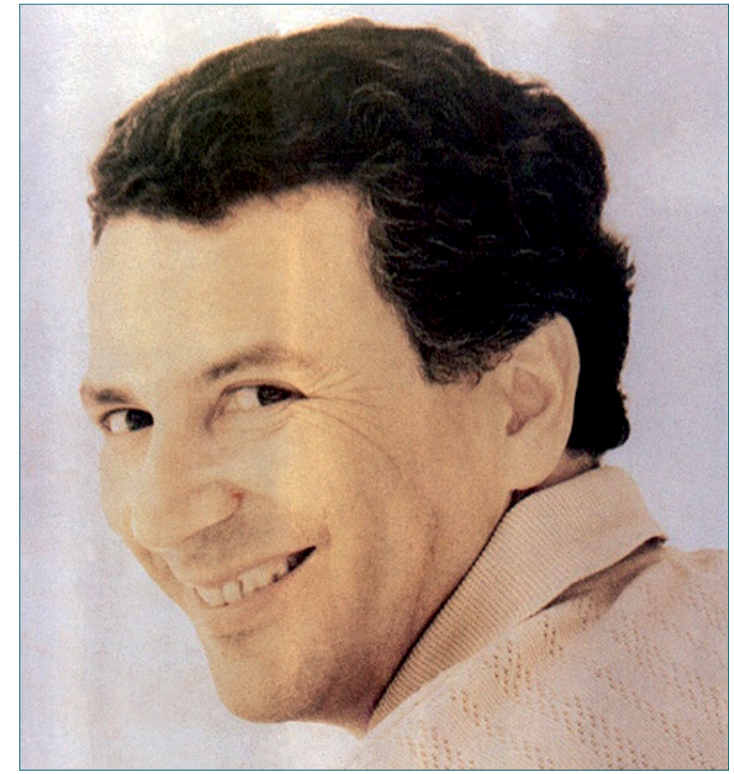

Figura 11. Max Arriagada Loyola (1934-1981).

logos.

Luego de la sensible muerte del Dr. Arriagada, lo sucedió en la jefatura del Servicio de Anestesiología y Pabellones Quirúrgicos uno de sus discípulos, el Dr. Guillermo Reyes, cargo que ocupó hasta el año 1986.

\section{La Posta Central en el inconsciente colectivo}

La presencia de "la Posta Central", en el imaginario popular de los chilenos, comenzó desde sus primeros años a tener un importante poder mediático, hasta llegar a ser máximo en los tiempos actuales. Este hecho podría quizás ser motivo de una profunda investigación sociológica y política; lo cierto es que opera como un barómetro tanto de la presión ambiental de la salud pública, como por extensión, de los cambios y transformaciones de nuestra propia sociedad.

Nacida hace 109 años, como ya se ha dicho de la imperiosa necesidad de auxiliar al prójimo afectado repentinamente por alguna dolencia traumática o de otra índole, ha llegado a entronizarse en el sentimiento popular como "algo propio, pero al mismo tiempo de todos".

Antes del surgimiento de los servicios de urgencia en las clínicas privadas, la Asistencia Pública era el lugar donde se atendía fundamentalmente a los beneficiaros, pero también a destacados políticos o empresarios. Siempre con el estilo de discriminación que los 
chilenos conocemos, existía un piso de pensionado, donde se hospitalizaba a personas destacadas, pero también al personal del hospital y a sus parientes directos.

La Asistencia Pública era sin duda un prestador de salud respetable y respetado. Los jefes de turno eran cirujanos de gran prestigio nacional e internacional y estaban orgullosos de pertenecer a la institución. Su habilidad quirúrgica y su capacidad para resolver graves problemas eran incuestionables, lo que los hacía ser respetados como líderes de opinión por cada turno, que constituía una verdadera familia.

A través de todos estos años, muchas señeras figuras de la medicina chilena han formado parte de la AP ("nuestra Posta Central"). Podemos mencionar a maestros de la Cirugía Chilena como los Drs. Emilio Salinas y Óscar Contreras o a un Maestro de la Anestesiología Chilena como el Dr. Ricardo Bustamante, quienes junto a cientos y probablemente miles de funcionarios clínicos, administrativos y de servicio, fueron creando una especie de mística que algunos han llamado "espíritu AP" y que consiste en motivarse con el dolor ajeno, ir más allá de sólo "cumplir con el trabajo", sirviendo, empatizando y solidarizando con el paciente de urgencia.

\section{El Servicio y su relación con la Sociedad de Anestesiología de Chile}

El Servicio de Anestesia, ha participado en forma regular de las actividades que organiza la Sociedad de Anestesiología de Chile, entre ellas cursos, talleres y particularmente el Congreso Chileno de Anestesiología que se realiza anualmente. Ésta se ha concretado de dos formas principales: a través de la participación de sus especialistas en conferencias y mesas redondas del programa oficial y a través de la presentación de trabajos de investigación clínica en la sección de temas libres. Respecto a esta actividad de investigación, es necesario destacar el premio recibido el año 1988 al servicio completo, por la presentación de once temas libres, que representaron un porcentaje sustantivo de la totalidad de trabajos presentados ese año, distinción que reconoció el volumen de trabajo realizado. Del mismo modo, es necesario destacar los premios "Profesor Luis Cabrera" recibidos los años 1994 y 1997 en los Congresos Chileno y Latinoamericano de Anestesia respectivamente, que distinguieron como los mejores trabajos del evento a los estudios clínicos realizados por los Drs. Ricardo Bustamante y Mauricio Ramos, reconocimiento que dio cuenta en estas oportunidades no del volumen, sino de la cali- dad de los mismos.

Es necesario, además, mencionar la participación de miembros del servicio en el Directorio de la Sociedad de Anestesiología de Chile, donde el Dr. Ricardo Bustamante se desempeñó como secretario en el bienio 1991-1993 durante la presidencia del Dr. Guillermo Lema y el Dr. Renato Chacón se desemepeñó como presidente en el bienio 2009-2011.

Por último, el mismo Dr. Ricardo Bustamante, participó activamente en la Revista Chilena de Anestesia, primero como Miembro del Consejo Editorial entre los años 1995 y 2007 y como Editor de la Revista entre los años 2008 y 2016.

\section{El desarrollo progresivo del Servicio de Anestesiología}

Durante la década de los 80, el desarrollo del Servicio de Anestesiología de la Posta Central significó la suma de nuevos especialistas que fueron ocupando los cargos disponibles tanto en horario diurno como de residencia y la incorporación de nueva tecnología que permitió ofrecer anestesias más complejas y más seguras.

La diversificación de las prestaciones quirúrgicas significó un proceso paralelo de las prestaciones anestésicas. El edificio de la Posta Central contaba con cuatro pabellones quirúrgicos centrales en el quinto piso, más un pabellón quirúrgico de uso exclusivo del Servicio de Quemados ubicado en el segundo piso. Además, existía un "pabellón de yesos" ubicado contiguo a las salas de hospitalización del Servicio de Traumatología, donde se realizaban procedimientos quirúrgicos menores y ortopédicos.

En horario diurno los cuatro pabellones centrales se encontraban habilitados y en ellos se realizaba cirugía traumatológica y cirugía general, esta última a cargo de un incipiente Servicio de Cirugía cuya misión era resolver cirugía semielectiva, el manejo de pacientes complejos como los de Unidad de Cuidados Intensivos y el seguimiento clínico de continuidad de los pacientes quirúrgicos en general.

Por su parte, el pabellón quirúrgico del Servicio de Quemados funcionaba solamente en horario hábil de mañana y tarde, a cargo de los cirujanos plásticos de dicho servicio, quienes realizaban el tratamiento integral de este grupo especial de pacientes. El Servicio de Quemados que durante muchos años estuvo a cargo del Dr. Mario Garcés, profesional de gran prestigio nacional e internacional, se convirtió en el centro de referencia nacional del paciente gran quemado. Este servicio contaba, además, con un residente que 
se encargaba de los pacientes hospitalizados y de los ingresos de nuevos pacientes en horario no hábil, profesional que podía requerir del anestesista de turno en caso de necesitar apoyo en el manejo clínico y en la realización de procedimientos invasivos.

En horario no hábil, dos de los pabellones centrales quedaban habilitados con personal técnico de enfermería, para la resolución de la patología quirúrgica de urgencia, a cargo del equipo de médicos de residencia.

Cada turno médico se encontraba conformado por seis cirujanos (el jefe de turno, dos primeros ayudantes y tres segundos ayudantes), un traumatólogo, un oftalmólogo, dos médicos internistas, dos anestesiólogos, un residente de quemados y dos médicos intensivistas. De los seis cirujanos el jefe de turno, un primer y un segundo ayudante subían a los pisos a pasar visita y luego a resolver los casos quirúrgicos de urgencia, conformando dos equipos con la participación de becados de cirugía provenientes de distintos programas de formación, que realizaban rotaciones por la Posta.

Complementariamente, era posible que uno de los dos pabellones disponibles fuera ocupado por el traumatólogo, para resolver fracturas que requirieran resolución de urgencia o por el oftalmólogo para el tratamiento de las urgencias oculares. Hasta la creación de la UTO (Unidad de Trauma Ocular) del Hospital Salvador, la Asistencia Pública fue el centro de referencia nacional de las urgencias oftalmológicas, llegando a contar con dos oftalmólogos por turno. Posteriormente, se incorporó un neurocirujano.

En resumen, en horario hábil el Servicio de Anestesiología distribuía sus profesionales en las operaciones programadas de cirugía general, traumatología y quemados y en horario no hábil en la cirugía general, traumatológica y oftalmológica que fuera considerada de urgencia. Todo ello implicaba, además, el manejo postoperatorio inmediato de los pacientes en la sala de recuperación anestésica, cuyo control, derivación y alta, eran de responsabilidad de los mismos profesionales.

Además de esta actividad clínica basal, tanto en horario diurno como nocturno, los anestesiólogos del servicio debían asumir labores complementarias. Una de ellas era realizar procedimientos invasivos en apoyo de otros profesionales del hospital entre los cuales lo más común eran la instalación de accesos vasculares (catéteres venosos centrales y líneas arteriales) y la intubación traqueal de pacientes graves en el Servicio de Urgencia, en la Unidad de Cuidados Intensivos y en el Servicio de Quemados.

Sumado a ello, era habitual que, al no contar con disponibilidad de cama en la Unidad de Cuidados Intensivos, los pacientes quirúrgicos graves tuvieran que cursar su posoperatorio inmediato en la Sala de Recuperación Anestésica, la cual para estos efectos convertía uno de sus cupos en una unidad intensiva supernumeraria, que con los escasos recursos de monitoreo, ventilación asistida y terapéuticos disponibles, era manejada por algunos de los anestesiólogos como residente.

Estos desafíos clínicos, que iban más allá de las responsabilidades propias del cargo, hacían imprescindible contar con dos anestesiólogos por turno, que cubrieran tan alta demanda.

El Dr. Carlos Reyes, también discípulo del Dr. Max Arriagada, sucedió al Dr. Guillermo Reyes en la jefatura del servicio a partir del año 1986, prolongando su jefatura hasta el año 1996. Durante su gestión, además de la incorporación de nuevos especialistas al equipo, tuvieron lugar varios progresos en los ámbitos administrativo, clínico y docente.

En lo administrativo, hasta el año 1986 el Dr. Guillermo Reyes ostentó el cargo de jefe de Servicios de Apoyo, bajo cuya responsabilidad se encontraba la gestión de servicios tan disímiles como Pabellones Quirúrgicos, Anestesiología, Radiología y Laboratorio Clínico. Evidentemente en la medida que cada uno de estos servicios crecía y se desarrollaba, era necesario diferenciar sus jefaturas, acorde con el aumento de sus respectivas complejidades, cosa que no se vio reflejada en el organigrama de la institución hasta varios años después. No obstante, el Dr. Carlos Reyes convenció a la autoridades del hospital de la necesidad de separar la gestión de jefatura de pabellones quirúrgicos y anestesiología, asunto que derivó en que al menos nominalmente él se desempeñó como jefe del Servicio de Anestesiología, en tanto la jefatura de Pabellones Quirúrgicos lo asumió la Dra. Eliana Hidalgo, momento a partir del cual ambas gestiones estuvieron separadas, asunto que como se señaló, con posterioridad fue refrendado en el organigrama en forma definitiva.

Desde el punto de vista clínico, los principales progresos tuvieron que ver con la incorporación de nuevos recursos en el manejo de los pacientes. Parte de estos avances se resumen en el siguiente listado:

- Incorporación de la ventilación mecánica de regla al acto anestésico: para este efecto, a las viejas máquinas de anestesia con que contaba el servicio, se adicionaron ventiladores mecánicos de presión y volumen, muchos de ellos equipos dados de baja y reciclados desde la Unidad de Cuidados Intensivos, que a través de ingeniosas conexiones otorgaban ventilación asistida a los pacientes so- 
metidos a anestesia general.

- Protocolización del monitoreo básico necesario para administrar anestesia general o regional: práctica que comenzó por el uso regular de monitoreo electrocardioscópico a través de antiguos osciloscopios y luego de monitores más sofisticados para la época, a lo que a fines de la década de los 80 se agregó el uso incipiente de presión arterial no invasiva, oximetría de pulso y capnografía, siendo este servicio el primero en Chile tanto en el ámbito público como privado en innovar en la integración de estos monitores a la práctica anestésica habitual.

- Ampliación del arsenal farmacológico a utilizar tanto en anestesia general como regional, con la incorporación de nuevos agentes inductores, bloqueadores neuromusculares y anestésicos locales.

- Finalmente, junto con la recuperación de la democracia en Chile en el año 1990, el Ministerio de Salud realizó una gran inversión en equipamiento anestésico, que incluyó la compra de modernas máquinas de anestesia y monitores, para la gran mayoria de los hospitales públicos del país. Esta inversión significó que los hospitales públicos y entre ellos la Posta Central, quedaran por un período de tiempo equipados con máquinas y monitores de una gama muy superior a aquellos con que contaban las instituciones privadas de salud.

\section{La docencia formal de posgrado en el servicio}

Como se mencionó previamente, el Dr. Max Arriagada desempeñó en el HUAP una labor docente similar a la que otros maestros realizaron en distintos hospitales del país. A través de esta política, muchos médicos se incorporaban a un servicio de anestesia donde al alero de uno de estos maestros, recibían la instrucción necesaria para convertirse en especialistas al cabo de algunos años de trabajo en dicho servicio. Es lo que se denominaba "formación en práctica", forma de especialización que fue preponderante previo a que ésta derivara principalmente a programas de formación de posgrado universitarios.

A partir de fines de la década de los 80 , la formación de posgrado en Chile comenzó a transformarse hacia programas estructurados de tres años; los oficiales bajo el alero de una universidad y otros no oficiales bajo el alero de algún servicio de la especialidad. Este fenómeno fue común a la gran mayoría de las especialidades y a muchos hospitales públicos del país, dentro de los cuales estuvo la "Posta Central", cuyo plan de formación regular de anestesiólogos se inició el año 1987, en que ingresó su primera promoción de cuatro residentes, fenómeno que no se detuvo más hasta el día de hoy.

Este programa evolucionó hacia un Programa Ministerial con sede en la Posta Central a partir del año 1993, y finalmente, se convirtió en el Programa de Postgrado en Anestesiología y Reanimación de la Universidad de Santiago de Chile a partir del año 1999, acreditándose por primera vez en el año 2013.

De las diferentes etapas formativas por las que ha transitado la docencia del servicio, han egresado un total de 189 anestesiólogos que se desempeñan como especialistas a lo largo de todo Chile. Del Programa Ministerial y luego Programa de Postgrado en Anestesiología de la Universidad de Santiago egresaron y siguen formando parte del servicio, completando 20 años de turnos de urgencia los Drs. Eliana Hidalgo, Mauricio Ramos, Renato Chacón, Andrea Moreno y Roberto Moreno. De ese mismo grupo, distinguidos colegas se han desplegado por distintos hospitales de Santiago y el resto de Chile: los Drs. Clara Luxoro, Marco Guerrero, Mariana Vargas, Juan Rivera, Luis Belmar, Carla Golppi, entre otros.

\section{El Servicio de Anestesia desde 1996 a 2019}

El Dr. Carlos Reyes renunció a la jefatura del servicio el año 1996, sucediéndolo el Dr. Víctor Hanna, quien se desempeñó en el cargo hasta el año 2007. Desde el 2007 al 2018 ejerció como jefa la Dra. Clara Luxoro y a partir de ese año lo hace el Dr. Rodrigo Figueroa (Figura 12).

Durante este período de 23 años, se han producido notables cambios en su estructura, progresos en su implementación y suma de nuevos desafíos. Se han agregado nuevas prestaciones que han requerido apoyo anestésico, entre ellas endoscopía intervencional y angiografía. El equipamiento ha sido renovado en varias ocasiones, trabajándose en este momento con máquinas de anestesia, monitores, bombas de infusión y otros equipos de última generación, tanto en los pabellones quirúrgicos como en la sala de recuperación anestésica. El arsenal farmacológico también ha sido renovado y a él se han agregado una gran cantidad de nuevos fármacos que permiten la administración de anestesia bajo estándares de alta complejidad y calidad. Un proceso similar se ha realizado con los insumos relacionados con el acto anestésico.

El número de anestesiólogos del servicio ha aumentado en forma progresiva en tanto aumenta la demanda clínica totalizando en la actualidad veinticinco especialistas, que cubren la actividad diurna y 
nocturna. Desde el año pasado ha aumentado el número de anestesiólogos de residencia a tres por turno, teniendo en cuenta la reciente inauguración de la Torre Valech y el aumento de la actividad quirúrgica que se ha proyectado en relación a ello.

La llegada de médicos del exilio, que acreditaron su especialidad en Chile, incorporó a la Dra. Paula Burchard, que se desempeñó por años como jefa de Pabellones Quirúrgicos, la Dra. Verónica Peña y el Dr. Daniel Reyes. Sin embargo, la gran mayoría de los especialistas que se han incorporado al equipo han sido formados en el mismo programa de posgrado, por lo cual su calidad técnica se encuentra avalada por el proceso docente que desarrolla el propio servicio.

El Hospital de Urgencia Asistencia Pública (HUAP), como se le denomina desde mediados de los años 90 , en que su nombre fue cambiado oficialmente (a pesar que todos la siguen reconociendo como la Posta Central) es considerado el Hospital de Urgencia y Trauma ícono del País, donde se atiende un número relevante de pacientes de urgencia y politraumatizados graves, lo cual significa un desafío importante para todos los servicios clínicos incluido el de anestesiología.

En febrero de 2019 se inauguró la Torre Valech (Figura 13), una ampliación de su planta física cuya obra gruesa fue financiada por Monseñor Sergio Valech a través de una donación de $\$ 7.000 .000 .000$, que fueron complementados por el Ministerio de Salud, para concretar su construcción definitiva, la cual tuvo un costo superior a los $\$ 40.000 .000 .000$. Este nuevo edificio de tres pisos, incorporó más de cincuenta boxes de atención de urgencia, veinticuatro boxes de unidad de cuidados intensivos y siete pabellones quirúrgicos, incluyendo uno híbrido para la realización de procedimientos angiográficos complejos, con equipamiento de última generación (Figuras 13 y 14).

\section{La pandemia de COVID-19}

El 11 de marzo de 2020, la OMS declaró oficialmente como "pandemia" el brote de coronavirus surgido en la ciudad de Wuhan, China. Ocho días antes, el 3 de marzo, se había reportado el primer caso en Chile. A partir de ese momento, la enfermedad evolucionó en el país con un mes de abril que mostró una meseta de contagios y hospitalización en unidades de cuidados intensivos y, posteriormente, con un mes de mayo en que ambas cifras aumentaron en forma dramática, sometiendo al sistema sanitario de salud chileno a una sobrecarga enorme, con miles de pacientes requiriendo ventilación mecánica y prolongados períodos de terapia intensiva, alcanzándose el peak de demanda durante la primera quincena de junio.

El 25 de mayo y ante las cifras de contagiados y de hospitalizados que crecían en forma incontenible, el Subsecretario de Redes Asistenciales Sr. Arturo Zúñiga declaraba ante los medios que el Ministerio de Salud había decidido "convertir a la Posta Central en el corazón de la batalla contra el coronavirus en la Región Metropolitana", a través de la reconversión de la totalidad de sus 250 camas en cupos para atender pacientes críticos con COVID-19. Si bien no se llegó a dicho nivel de conversión de camas, la Posta Central sí se constituyó en el epicentro sanitario de la pandemia.

Durante dicho período, se duplicó los cupos de atención de pacientes en el servicio de urgencia, se multiplicó por cinco la capacidad de ventilación mecánica desde los 24 cupos de UCI originales a más de 100 , repartidos en unidades de cuidados intermedios, salas de medicina interna y cirugía adaptadas, cupos en la Unidad de Recuperación Anestésica y pacientes ventilados con máquinas de anestesia en pabellones quirúrgicos. En el período de máxima demanda, hubo

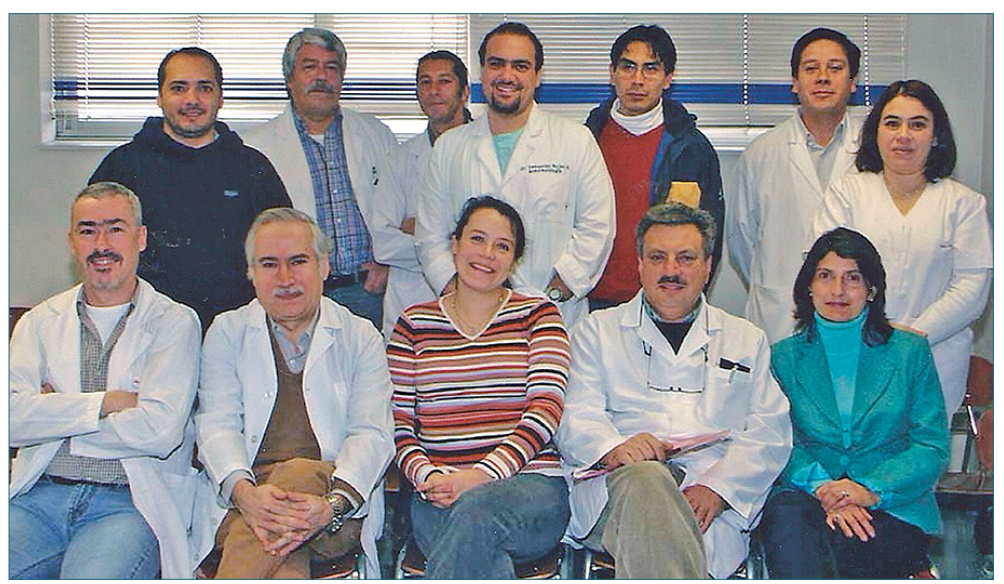

Figura 12. Algunos miembros del Servicio de Anestesiología del HUAP en el año 2005. Primera fila (sentados): Dr. Renato Chacón, Dr. Ricardo Bustamante, Dra. Carla Golppi, Dr. Víctor Hanna (entonces jefe de Servicio) y Dra. Verónica Peña. Segunda fila (de pie): Dr. Roberto Moreno, Dr. Guillermo Reyes (jefe hasta 1986), Dr. Daniel Leiba (becado), Dr. Sebastián Rojas (becado), Dr. Miguel Ángel Núñez (becado), Dr. Mauricio Ramos y Dra. Clara Luxoro (jefa hasta el 2018). 


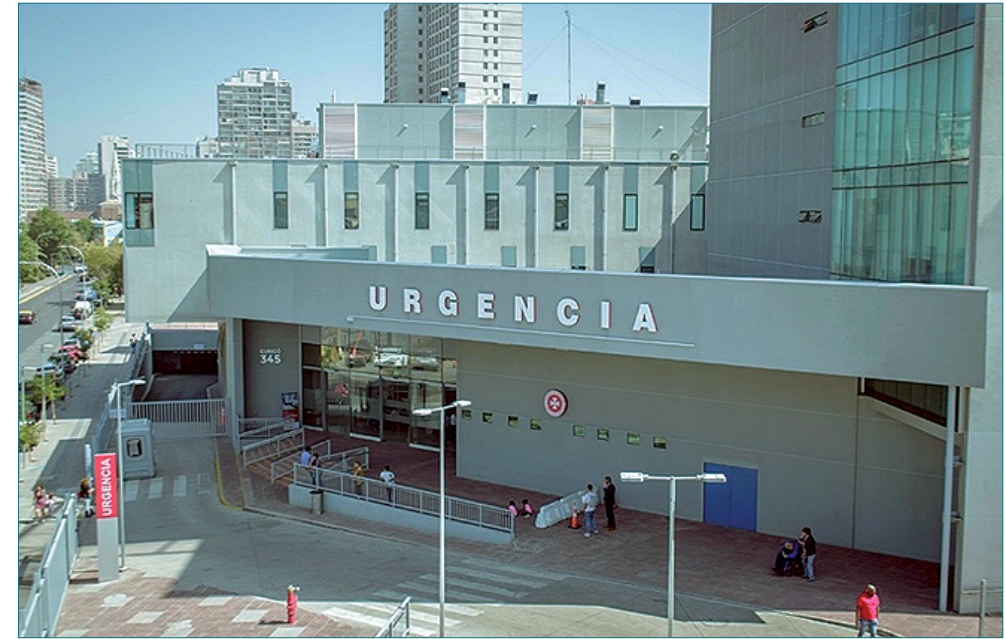

Figura 13. Edificio de la Torre Valech, donde funcionan a partir de 2019 los Servicios de Urgencia, Unidad de Cuidados Intensivos y Pabellones.

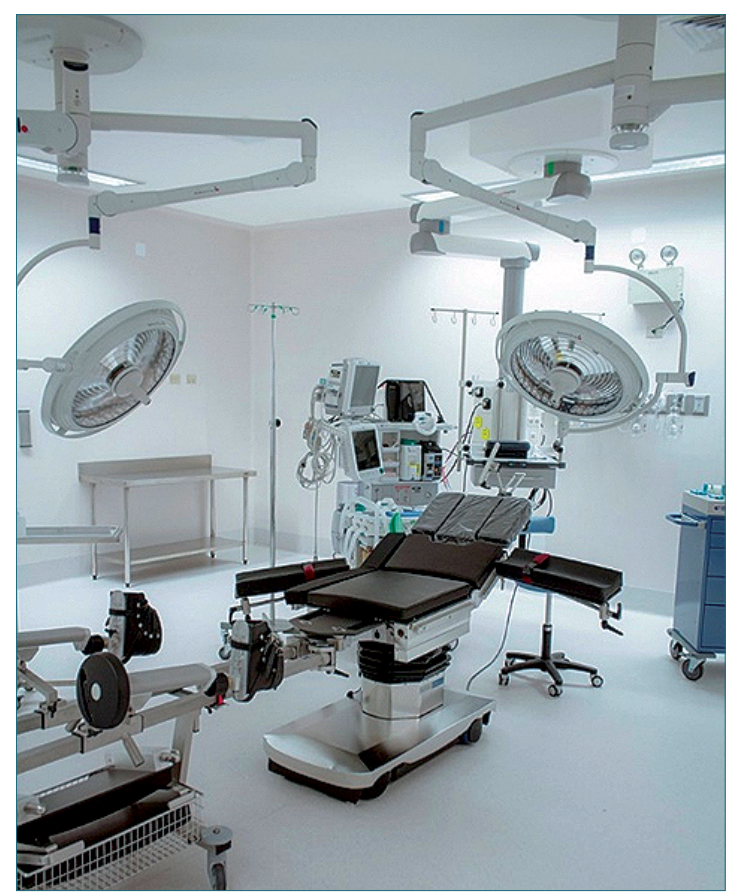

Figura 14. Pabellón de la Torre Valech, plenamente equipado para la cirugía de urgencia.

cerca de 90 pacientes en ventilación mecánica invasiva y más de 50 en ventilación mecánica no invasiva o con sistemas de oxigenoterapia compleja como sistemas Helmet o cánulas nasales de alto flujo.

Durante la crisis, los miembros del Servicio de Anestesiología, además de mantener su función ba- sal de provisión de anestesia para los pacientes que requerían cirugías de urgencia (muchos de ellos contagiados por coronavirus), participaron en el manejo de pacientes críticos en ventilación mecánica en una de las unidades habilitada para tal efecto, manejaron pacientes en ventilación mecánica (por COVID-19 u otra causa) en la Unidad de Recuperación Anestésica y ventilaron pacientes en pabellones quirúrgicos, primero con máquinas de anestesia y luego con ventiladores convencionales, aportando así al enfrentamiento de la emergencia sanitaria.

El 7 de agosto de 2020 con motivo del aniversario número 109 del hospital, se realizó un recuento de la atención brindada durante la pandemia, que daba cuenta que de los 27.405 pacientes hospitalizados por COVID-19 en Chile y de los 19.599 de la Región Metropolitana hasta esa fecha, 2.259 lo habían hecho en la Posta Central, representando $8,24 \%$ de la cifra nacional y $11,52 \%$ de la cifra regional. Es decir, efectivamente el hospital se constituyó en uno de los epicentros del manejo de la pandemia.

Tan importante como lo anterior, es que tratándose de una población en que cerca del $85 \%$ de los pacientes cumplieron criterios de gravedad, fellecieron 247 de ellos, representando una letalidad del $10,93 \%$. Sin desconocer el drama que representa cada una de esas muertes, esa cifra de letalidad se constituye en una cifra bajísima en comparación con datos internacionales y de otras instituciones del país, que refleja el esfuerzo conjunto de todos los funcionarios de la institución, por una vez más, ser parte del eje estructurador de la atención de urgencia en Chile en situaciones de emergencia. 


\section{El espíritu}

Han pasado los años, ha cambiado la medicina, han cambiado nuestros hospitales. Sin embargo, entre estos y muchos otros cambios, pienso que persiste algo inmutable, que es fundamental en esta institución... el llamado "espíritu AP", para algunos difícil de definir, sobre todo en nuestra actualidad tan individualista y un tanto egoísta, pero que no es más que ponerse en el lugar del otro, empatizar con él, cumpliendo más allá de la obligación con la labor asistencial. Así entonces, es fácil comprender el por qué la Posta Central, está en la mentalidad de la mayoría de los chilenos.

\section{Referencias}

1. El dolor ajeno (Reinaldo Martinez - 1991).

2. Historia de la medicina chilena (Ricardo Cruz Coke - 1998).

3. Organización del trabajo hospitalario (Ignacio González G.1946).

4. Ernesto Greves S. (Los antiguos hospitales, médicos, cirujanos y farmacéuticos - 1933).

5. Jornadas de la historia de la medicina chilena (Amador Negme - 1987).

6. El guardián de la salud (Leo Manfred - 1949).

7. Archivos documentación (Diario
El Mercurio de Valparaíso 1848).

8. Revista de la Asistencia Pública (1970)

9. Revista "Vida Médica" del Colegio Médico de Chile - Julio 1958.

10. Epistolario (A- Bulnes - 1967).

11. Archivo fotográfico HUAP.

12. Archivo Revista de la Beneficencia Pública (1922).

13. Álvarez JP. Francisco Javier Villanueva: La primera administración de una anestesia general en Chile. Rev Med Clín Las Condes 2013; 2: 320-23. https://doi.org/10.1016/S07168640(13)70167-6
14. Venturini H. Historia de la Anestesia en Sudamérica, Accesible en: http://files.sid.cu/anestesiología/files/2012/2013/03/ anestesia-sudamérica.pdf.

15. Álvarez JP. Dr. Ernesto Frías Meneses. El primer anestesiólogo chileno. Rev Med Clín Las Condes 2017; 28: 803-11. https://doi.org/10.1016/j.rmclc.2017.10.005

16. Bustamante R. Maestros de la Anestesiología Chilena. Rev Chil Anest 2015; 39; 251-3.

17. Muñoz E,Neumann $H$, Morales M. Los orígenes de la anestesia en Chile. Revisión Histórica. Rev Chil Cir 2000; 52; 305-12. 